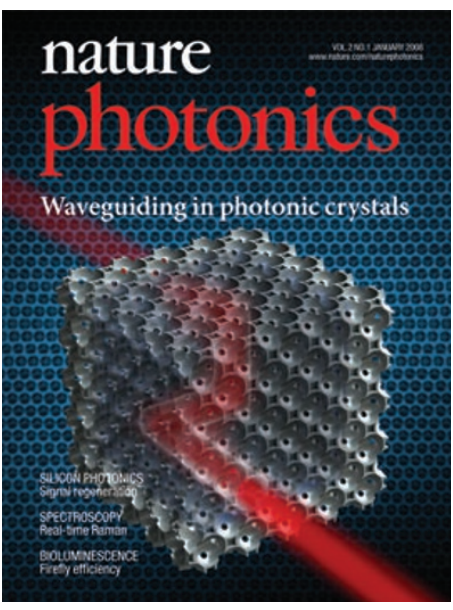

\section{Cover story}

Vol.2 No.1 January 2008

\begin{abstract}
Photonic crystals, artificially engineered nanoscale structures that can manipulate the flow of light, show great promise for building sophisticated optical circuitry that can route, filter, store or suppress optical signals. However, fabricating such circuitry presents a great challenge as defects need to be carefully incorporated into the photonic-crystal structure with great precision. Although this has been accomplished for two-dimensional designs that confine light in a plane, it is still an ongoing challenge for so-called complete-bandgap materials, where the defects need to be embedded into a three-dimensional structure. In this issue, Paul Braun and colleagues report the introduction of defects into a silicon three-dimensional photonic crystal by using a technique called two-photon polymerization. The result is waveguides that guide near-infrared light around sharp corners. [Article p52; News \& Views p9]
\end{abstract}

\section{SILICON REGENERATION}

The ability to regenerate weak and distorted optical data is vital for maximizing the performance and transmission span of modern communication systems. Unfortunately, present regenerators have to convert the optical data into electronic signals, process it and then convert it back into the optical domain for retransmission a costly and inconvenient approach. Now, however, Alexander Gaeta and co-workers from Cornell University in the USA have developed an all-optical scheme that can perform complete regeneration (amplification, retiming and reshaping of optical data bits) using silicon waveguides that have nanoscale dimensions. The technique exploits a well-known technique in nonlinear optics, called four-wave mixing, to transfer noisy and degraded data bits from an incoming light beam and convert them into high-quality, clean data bits carried on a second light beam at a different wavelength. The beauty of the approach is that it does not require any electronic circuitry, and the tiny size and silicon composition of the set-up give it the potential for integration into optical chips in the future. The team's experiments took place at a wavelength of $1,550 \mathrm{~nm}$, within the region at which the world's optical networks operate.

[Letter p35; News \& Views p12]

\section{BACK ON TRACK}

Involving over 30,000 optical components, 430 tonnes of ultrahigh-purity glass and 192 high-energy laser beams, there is no doubt that the construction of the National Ignition Facility (NIF) in the USA is a gigantic undertaking. Based in California and the size of two football fields, the world's largest laser facility is being built to attempt laser-induced nuclear fusion. The idea is that 192 UV laser beams will 'dump' around $500 \mathrm{TW}$ of power into a 2 -mm-sized target containing a mixture of deuterium and tritium and create conditions of sufficiently high temperature and pressure to induce nuclear fusion. Despite initial problems with its budget and time schedule, which have made the facility a rather controversial topic, the $\$ 3.5$ billion project is now firmly back on track according to its director Ed Moses. Moses adds that 104 of the 192 laser beams are now operational. The first fusion tests at the NIF are expected to begin in 2010. [Profile p3]

\section{BACK TO THE LIGHTNING BUG}

Fireflies are famous for their

bioluminescence - a chemical reaction that generates a yellow glow that can be seen with the naked eye on balmy summer evenings. For the past 50 years, the efficiency of this light generation has been based on a quantum-yield measurement of $88 \%$. However, it now seems this figure is incorrect. Yoriko Ando and colleagues from the University of Tokyo describe how they used a specially developed total-photon-flux spectrometer to measure a new value of $41 \%$, around half the original estimate. The results not only overturn the widely accepted value for the efficiency of the light generation, but also cast doubts over the mechanism behind the colour change seen in fireflies.

[Letter p44; News \& Views p8; Interview p60]

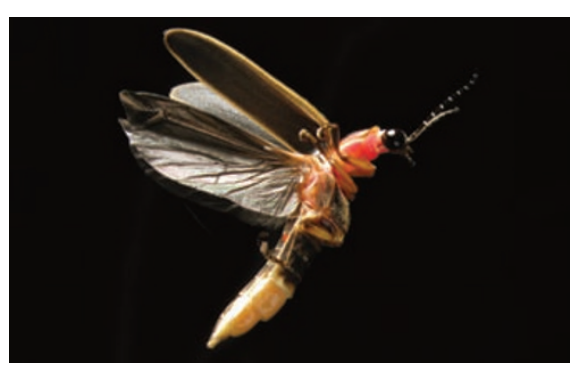

New results on firefly bioluminescence overturn previously accepted views on its efficiency.

\section{BIOMECHANICAL BREAKTHROUGH}

Biomechanical studies of the eye and other body parts could be about to become much easier to perform, thanks to the demonstration of a high-resolution noncontact technique that exploits Brillouin imaging. In this issue, Seok Hyun Yun and Giuliano Scarcelli report the construction of an efficient confocal Brillouin microscope that exploits a fully parallel spectrometer based on a virtually imaged phase array. The microscope is able to make high-resolution dynamic measurements of the mechanical properties of biological tissue and other materials. To demonstrate its capabilities, Yun and Scarcelli make the first in situ mechanical measurements of the crystalline lens in a mouse eye, as well as monitoring dynamic changes while curing an optical polymer. In the future, they have the opinion that the technique could be extended to the cellular scale to suit in vivo measurements.

[Letter p39; News \& Views p13]

\section{REAL-TIME ANALYSIS}

Spectroscopy is a valuable and attractive tool for analysing processes. However, it is extremely challenging to perform in real time for dynamic events. One of the most promising approaches so far is so-called wavelength-time spectroscopy, which chirps the spectrum and maps wavelengths into time so that spectra can be quickly captured by a single-shot oscilloscope. However, spectral measurements with high resolution incur a large optical loss. Daniel Solli and colleagues from the University of California, Los Angeles, may now have an answer to the problem. In this issue, they show how they used distributed amplification to overcome the loss and perform real-time Raman spectroscopy. The authors are of the opinion that the approach could suit dynamic biomolecular analysis and be used with microscopy. [Letter p48] 